AperTO - Archivio Istituzionale Open Access dell'Università di Torino

\title{
Ultra high energy photons and neutrinos with JEM-EUSO
}

\section{This is a pre print version of the following article:}

Original Citation:

Availability:

This version is available http://hdl.handle.net/2318/1653124

since 2017-11-25T12:47:03Z

Published version:

DOI:10.1007/s10686-013-9353-2

Terms of use:

Open Access

Anyone can freely access the full text of works made available as "Open Access". Works made available under a Creative Commons license can be used according to the terms and conditions of said license. Use of all other works requires consent of the right holder (author or publisher) if not exempted from copyright protection by the applicable law. 


\title{
Ultra High Energy Photons and Neutrinos with JEM-EUSO
}

\author{
The JEM-EUSO Collaboration
}

Received: date / Accepted: date

\begin{abstract}
Ultra high energy photons and neutrinos are carriers of very important astrophysical information. They may be produced at the sites of cosmic ray acceleration or during the propagation of the cosmic rays in the intergalactic medium. In contrast to charged cosmic rays, photon and neutrino arrival directions point to the production site because they are not deflected by the magnetic fields of the Galaxy or the intergalactic medium. In this work we study the characteristics of the longitudinal development of showers initiated by photons and neutrinos at the highest energies. These studies are relevant for development of techniques for neutrino and photon identification by the JEM-EUSO telescope. In particular, we study the possibility of observing the multi-peak structure of very deep horizontal neutrino showers with JEM-EUSO. We also discuss the possibility to determine the flavor content of the incident neutrino flux by taking advantage of the different characteristics of the longitudinal profiles generated by different type of neutrinos. This is of grate importance for the study of the fundamental properties of neutrinos at the highest energies. Regarding photons, we discuss the detectability of the cosmogenic component by JEM-EUSO and also estimate the expected
\end{abstract}

JEM-EUSO Collaboration

for full author list see first paper of this volume

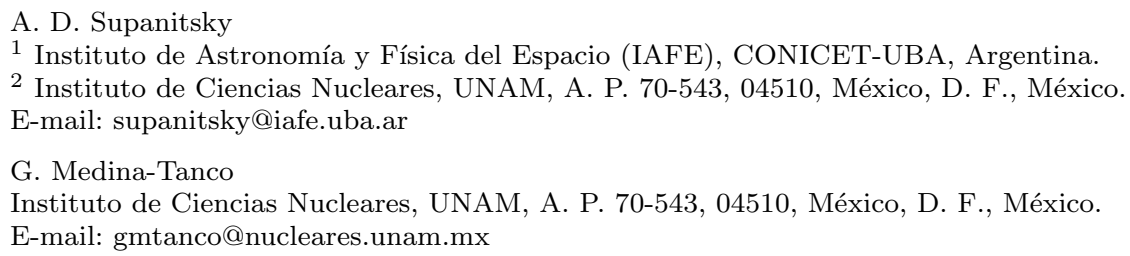


upper limits on the photon fraction which can be obtained from the future JEM-EUSO data for the case in which there are no photons in the samples.

Keywords Neutrinos · Photons · Space observation

\section{Introduction}

In the last few years the understanding of the cosmic rays at the highest energies has been significantly advanced due largely to the significant amount of high-quality data recorded by the Pierre Auger Observatory. However, the origin and composition of these energetic particle remains unknown. A promising approach to help unravel this mystery involves multimessenger observations. High energy photons and neutrinos play a fundamental role in these types of studies. One of the advantages of the observation of neutral particles like photons and neutrinos is that their trajectories are not deflected by the Galactic and intergalactic magnetic fields and thus point back to the production site.

Ultra high energy $\left(E \gtrsim 10^{18} \mathrm{eV}\right)$ photons [1-3] and neutrinos [4-14] can be generated as a by-products of the interaction of cosmic rays during their propagation through the intergalactic medium. They can also be produced by cosmic ray interactions in the acceleration sites [15-18]. Furthermore, they can be generated in top-down scenarios involving the decay of super heavy relic particles or topological defects [19-21] though this is disfavored by current data. Very recently, the IceCube Collaboration reported an observation of 28 neutrino candidates in the energy range $10 \mathrm{TeV}<E_{\nu}<1 \mathrm{PeV}$ [22]. This constitutes a 4.3 sigma excess over the expected background from atmospheric neutrinos, suggesting that the era of cosmic neutrino astronomy may be close at hand.

The flavor content of the astrophysical neutrino flux is a function of the neutrino energy [23-25]. One of the main mechanisms that causes the flavor ratio to change is based on the fact that pions and muons produced in the sources lose their energy because of the interaction with the ambient magnetic field. The pion decay time is shorter than the corresponding one to the muon, and then, at sufficiently high energies, the probability of pion decay, prior to significant energy loss, is larger than the corresponding one to the muon. As a consequence, the flavor ratio at the source changes from $\Phi_{\nu_{e}}: \Phi_{\nu_{\mu}}: \Phi_{\nu_{\tau}}=1$ : $2: 0$ to $\Phi_{\nu_{e}}: \Phi_{\nu_{\mu}}: \Phi_{\nu_{\tau}}=0: 1: 0$. The transition between theses two regimes depends on the characteristics of the source. Therefore, the determination of the flavor content of the flux is of great importance for the understanding of the physical processes that take place in the source. The flavor information can also be used to study the more fundamental properties of neutrinos and to identify physics beyond the standard model (see, for instance, Refs. [26-28]).

Here we consider the potential to observe ultra high energy neutrinos and photons using orbital detectors like JEM-EUSO. The identification of events initiated by these primary particles is based on the unique characteristics of the longitudinal profiles of the showers that they generate in the atmosphere. In this work we study the characteristics of the electron and tau neutrino induced 
horizontal air showers that develop very deep in the atmosphere. We discuss the possibility to discriminate between electron and tau neutrinos by using the multi-peak structure of the longitudinal profiles present in these types of events. We also evaluate the response of the JEM-EUSO telescope to these types of showers and discuss the possibility to discern the predicted multipeak structure in the data. Furthermore, we study the possibility to identify photons using the atmospheric depth at which showers reach their maximum size as the discrimination parameter. Finally, we estimate the number of photon events expected by JEM-EUSO for an optimistic astrophysical model and also calculate the upper limit on the photon fraction in the integral cosmic ray flux which can be obtained for the case in which no photons are observed.

\section{Neutrinos}

\subsection{Neutrino-nucleon interaction}

High energy neutrinos that propagate in the Earth's atmosphere can interact with the protons and neutrons in air molecules. There are two possible channels for this interaction, charged current (CC) and neutral current (NC) :

$$
\begin{array}{ll}
\mathrm{CC}: & \nu_{\ell}+N \rightarrow \ell+X, \\
\mathrm{NC}: & \nu_{\ell}+N \rightarrow \nu_{\ell}+X .
\end{array}
$$

Here $N$ is a nucleon (proton or neutron), $\nu_{\ell}$ is a neutrino of the family $\ell$, where $\ell$ is the corresponding lepton, and $X$ denotes the hadronic products of the interaction. At the level of the quark-parton model, the entire hadronic state of a deep inelastic scattering may be viewed as the fragmented product of a scattered quark and the proton remnant. The major uncertainty on the differential cross section at the energies considered here comes from the unknown behavior of the parton distribution functions (PDFs) at very small values of the parton momentum fraction $x$ [29].

In this work, the simulation of the neutrino-nucleon interaction is performed by using the PYTHIA code [30] linked with the library LHAPDF [31] which allows for different extrapolations of the PDFs. PYTHIA is an event generator, intended for high-energy processes with particular emphasis on the detailed simulation of quantum chromodynamics (QCD) parton showers and the fragmentation process. Figure 1 shows the distribution function of the energy fraction carried by the electron as a result of a CC interaction of a $10^{20}$ $\mathrm{eV}$ electron neutrino with a proton. The set of PDFs used for the simulation is CTEQ66 [32]. The distribution function of the energy fraction carried by the leading particle after a $\mathrm{CC}$ or $\mathrm{NC}$ interaction depends on the primary energy of the incident neutrino. Figure 2 shows the mean value of the energy fraction of the leading electron neutrino generated in an electron neutrino-proton $\mathrm{CC}$ interaction as a function of the electron neutrino energy. The energy fraction carried by the electron increases with the energy of the incident electron neutrino reaching values close to 0.82 at $E_{\nu_{e^{-}}}=10^{20} \mathrm{eV}$. 


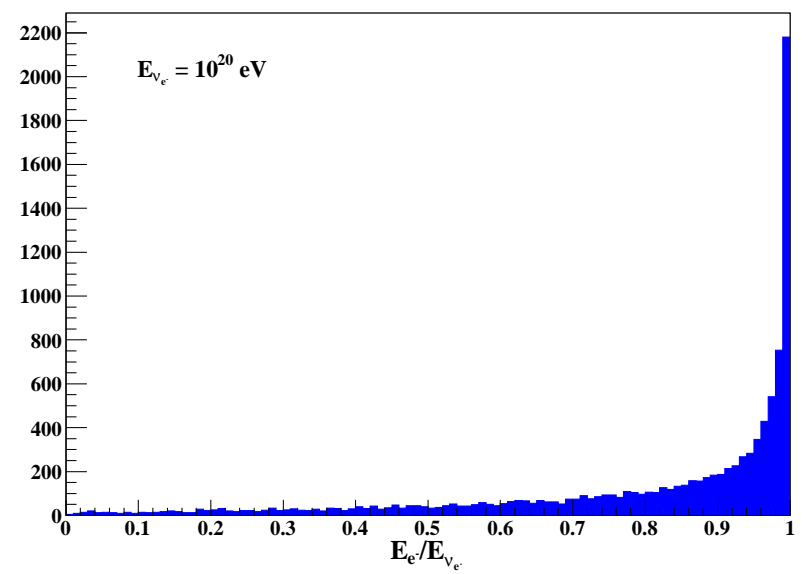

Fig. 1 Distribution of the energy fraction carried by the electron in an electron neutrinoproton charged current interaction. The electron neutrino energy is $E_{\nu}=10^{20} \mathrm{eV}$ and PYTHIA with the CTEQ66 set of PDFs are used for the simulation.

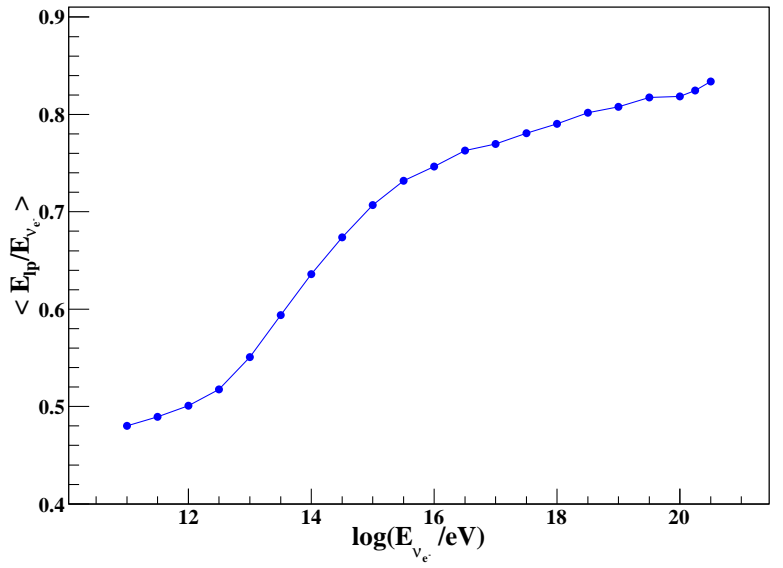

Fig. 2 Average energy fraction taken by the electron in an electron neutrino-proton charged current interaction as a function of the neutrino energy. PYTHIA with the CTEQ66 set of PDFs is used for the simulation.

\subsection{Characteristics of neutrino showers}

Neutrinos can initiate atmospheric air showers when they interact with the nucleons of air molecules. The CC interactions are the most important for space-based observations because in $\mathrm{NC}$ interactions the majority of the energy is carried off by a secondary neutrino which can only produce an observable air shower if it suffers a subsequent CC interaction. The potential to observe showers produced by the hadronic component resulting from the NC interaction depends on the energy threshold of the telescope. In this work, only showers initiated by CC interactions are considered. Note that the probability 
that a neutrino interacts in the atmosphere increases with the zenith angle because of the increase of the density of target nucleons.

Electron neutrino showers are simulated following Ref. [33]. As mentioned before, the charged current neutrino-nucleon interaction is simulated by using PYTHIA with the CTEQ66 set of PDFs. The secondary particles produced in the interaction are used as input in the program CONEX [34] (v2r2.3) in order to simulate the shower development. The high energy hadronic interaction model used for the shower simulations is QGSJET-II [35].

Because the mean free path of neutrinos propagating in the atmosphere is very large, they can interact very deeply, after traversing a large amount of matter. An orbital detector like JEM-EUSO can detect horizontal showers that do not hit the ground. In particular, horizontal neutrinos can interact at higher altitudes producing a shower more easily observable from orbit. Figure 3 shows the energy deposit as a function of $X-X_{0}$, where $X$ is the atmospheric depth and $X_{0}$ is the atmospheric depth of the first interaction point for horizontal electron neutrino showers of $E_{\nu}=10^{20} \mathrm{eV}$. The first interaction points are shown at various altitudes for shower trajectories starting at the vertical axis of the JEM-EUSO telescope in Nadir mode.
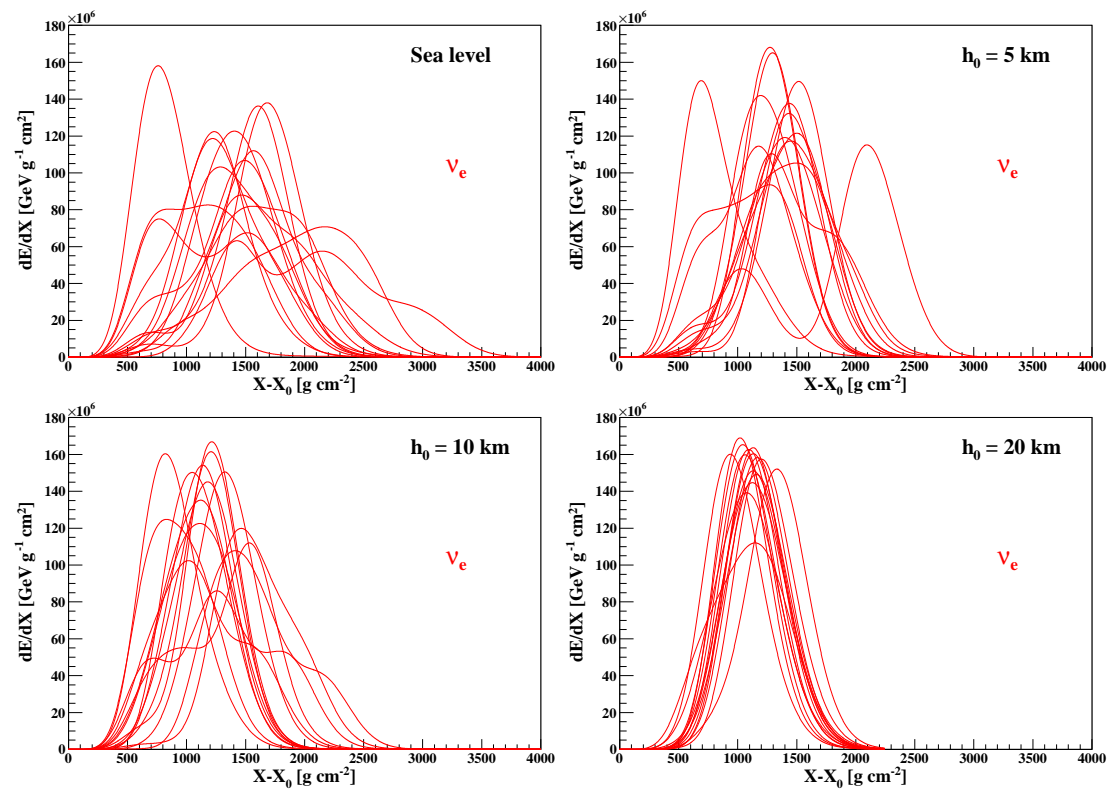

Fig. 3 Horizontal electron neutrino showers of $E_{\nu}=10^{20} \mathrm{eV}$ at four different altitudes.

The profiles corresponding to lower altitudes exhibit very broad distributions, large fluctuations, and may possess several peaks. This behavior is due to the Landau and Pomeranchuk [36,37], Migdal [38] (LPM) effect, which is very important for electromagnetic particles in dense regions of the atmosphere; in this case, for the electrons which carry on average $82 \%$ of the parent neu- 
trino energy. It can also be seen that as the altitude increases the fluctuations are reduced and, on average, the profiles become narrower. This is a result of the LPM effect becoming progressively less important with increasing altitude due to the decrease in atmospheric density. Figure 4 shows the probability of producing profile with a given number of peaks, $N\left(X_{\text {max }}^{i}\right)$. As the altitude increases the probability of finding a shower with more than one peak becomes negligible.

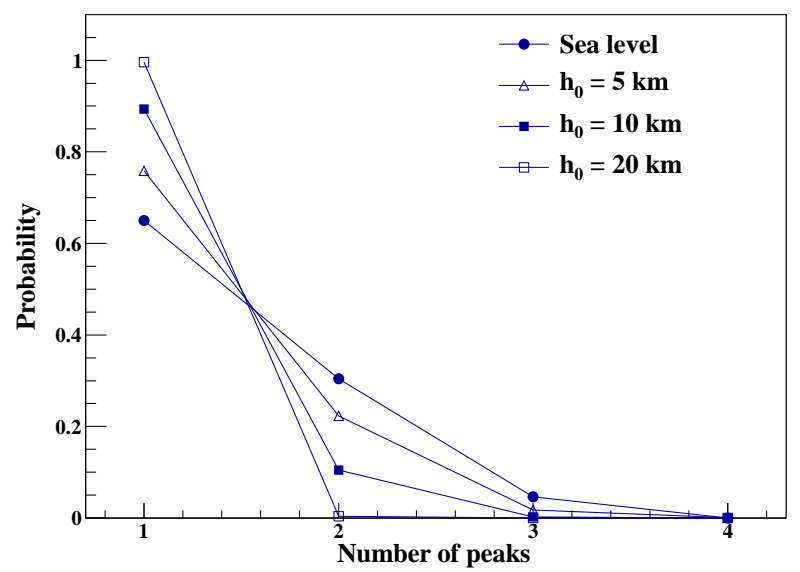

Fig. 4 Probability of an electron neutrino shower to have $N\left(X_{\max }^{i}\right)$ maxima in the longitudinal profile of the shower, for $E_{\nu}=10^{20} \mathrm{eV}$ for horizontal showers at different altitudes.

In the case of tau neutrinos, the hadronic component $X$ initiates a low energy shower immediately after the $\mathrm{CC}$ interaction (first bang), while the generated tau lepton propagates through the atmosphere almost without interacting and then, after a given distance, decays. The particles produced in the tau decay initiate a more energetic shower than the first one, producing a second bang. There are several tau decay channels which we classify in three categories: [40],

- Electromagnetic channel: $\tau \rightarrow \nu_{\tau}+e^{-}+\nu_{e^{-}}$.

- Hadronic channels: $\tau \rightarrow \nu_{\tau}+X$, where $X$ can comprise pions, kaons, etc.

- Muonic channel: $\tau \rightarrow \nu_{\tau}+\mu^{-}+\nu_{\mu}$.

The branching ratios of the electromagnetic, hadronic, and muonic channels are $b_{e m}=0.18, b_{h}=0.645$, and $b_{\mu}=0.175$, respectively. The tau neutrino showers in the muonic channel are difficult to observe because of the large decay length of muons at the energies considered. Consequently we consider here only the electromagnetic and hadronic channels, in which case $\sim 78 \%$ of the showers are of hadronic origin and $\sim 22 \%$ are of electromagnetic origin.

The simulation of tau neutrino showers is performed following Ref. [39]. The $\mathrm{CC}$ tau neutrino-nucleon interaction and the production of the corresponding tau lepton are simulated using PYTHIA with the CETQ66 set of PDFs. The 
decay of the tau is simulated with the program TAUOLA [40]. The resulting particles are used as input in CONEX with QGSJET-II in order to simulate the shower development.

Although the tau decay length is quite large at the energies under consideration, $\lambda_{\tau} \cong 4900 \times\left(E_{\tau} / 10^{20} \mathrm{eV}\right) \mathrm{km}$, there is a non zero probability to have a tau decay (i.e. the second bang) in the central region of the JEM-EUSO field of view. In particular, considering horizontal showers at sea level, the ratio between the number of events for which the tau decays in the central region of the JEM-EUSO field of view and the number of events corresponding to electron neutrinos interacting at the same point decreases from $\sim 1$ at $E_{\nu}=10^{18}$ $\mathrm{eV}$ to $\sim 0.13$ at $E_{\nu}=10^{19.75} \mathrm{eV}$ (see Ref. [39] for details of the calculation). In the subsequent analysis just the second bang is considered.

Figure 5 shows the longitudinal profiles for horizontal tau neutrino showers of $E_{\nu_{\tau}}=10^{20} \mathrm{eV}$ for which the point where the tau decays is placed at sea level on the vertical axis of the JEM-EUSO telescope in nadir mode. As for the case of electron neutrino showers, the multiple peak structure present in the profiles is caused by fluctuations introduced by the LPM effect. As expected, tau neutrino showers are less affected by the LPM effect than electron neutrino showers. This is because $\sim 78 \%$ of the detectable tau neutrinos showers are hadronic.

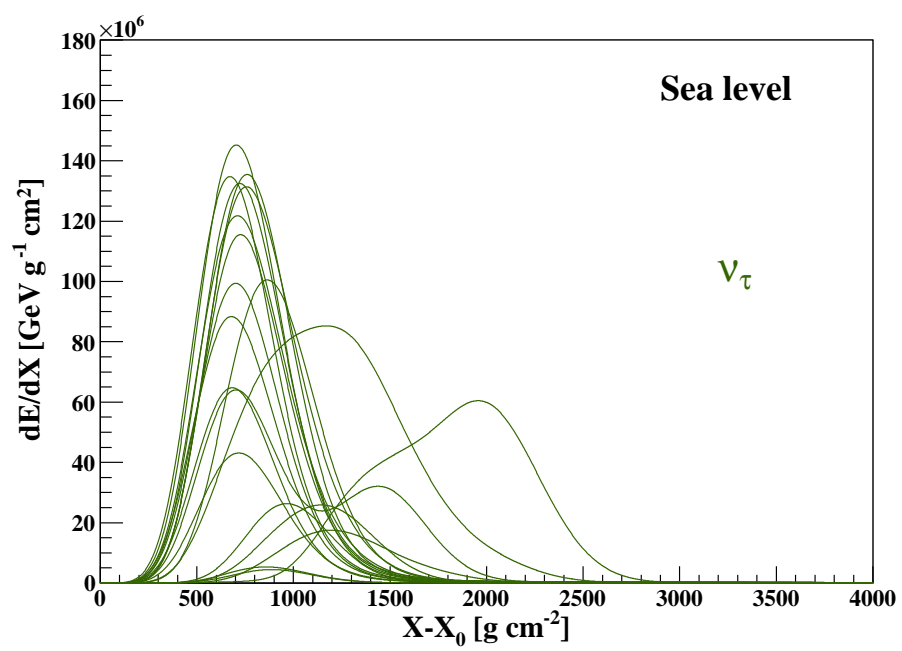

Fig. 5 Horizontal tau neutrino showers of $E_{\nu_{\tau}}=10^{20} \mathrm{eV}$ at sea level.

The number and positions of peaks in a given simulated profile are determined following the procedure described in Ref. [33]. Figure 6 shows the distribution of the position of the first peak, $X_{\text {max }}^{1}$, relative to the first interaction point, $X_{0}$, for electron and tau neutrino horizontal showers of $E_{\nu}=10^{20}$ $\mathrm{eV}$ in which the first interaction point is at sea level and on the vertical axis of JEM-EUSO in nadir mode. Note that the distribution function of 
$\Delta X_{\max }^{1}=X_{\max }^{1}-X_{0}$ for electron neutrino showers is bi-valued and its first peak is located at $\Delta X_{\max }^{1} \sim 800 \mathrm{~g} \mathrm{~cm}^{-2}$, while the second one is centered at $\Delta X_{\max }^{1} \sim 1500 \mathrm{~g} \mathrm{~cm}^{-2}$. The first peak is related to the development of the hadronic component of the cascade, whereas the second one reflects mainly the electromagnetic components of the shower [33]. As expected, the distribution function of $\Delta X_{\max }^{1}$ for tau neutrino showers is also bi-valued but the hadronic peak is much more important.

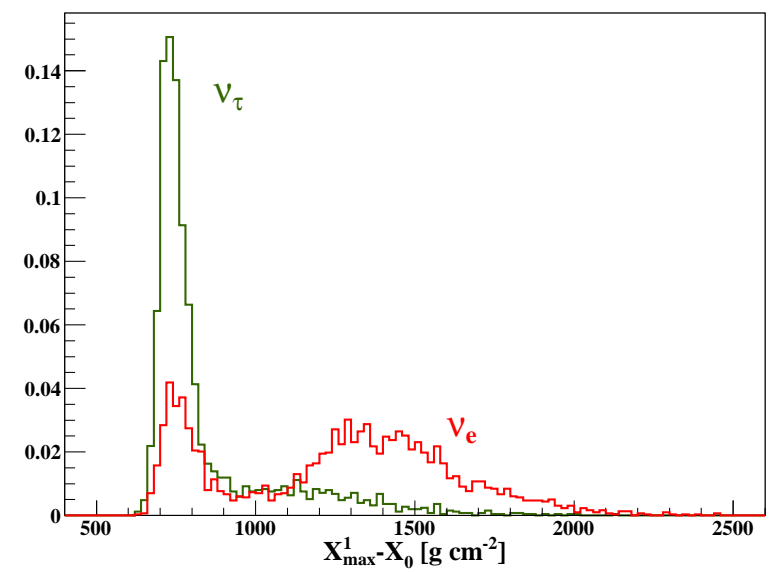

Fig. 6 Distribution of the position of the first peak (in grammage) for electron and tau neutrino showers whose first interaction point is at sea level and on the vertical axis of JEMEUSO in Nadir mode. The neutrino energy is $E_{\nu}=10^{20} \mathrm{eV}$. The histograms are normalized to unity.

Figure 7 shows the probability to find a profile with $N\left(X_{\max }^{i}\right)$ peaks. As expected, the profiles corresponding to electron neutrino showers have a larger probability to have more than one peak than profiles from tau neutrino showers. In particular, the probability to have just one peak for electron neutrino showers of $E_{\nu}=10^{20} \mathrm{eV}$ is $\sim 0.67$, whereas for tau neutrino showers it is $\sim 0.98$.

In Ref. [39] it is shown that the fraction of showers with just one peak, $f_{1}=n_{1} / N$, can be used as a parameter to discriminate between tau and electron neutrinos. Even with a sample with small number of events $(N \gtrsim 10)$ it is possible to say something about the flavor content of the incident neutrino flux.

Muon neutrino showers can be initiated by the electrons generated in the dominant decay channel of the muons produced in the CC interaction. However, these showers are difficult to observe because of the very large decay length of the muon at such high energies, $\lambda\left(E_{\mu}\right) \cong 6.23 \times 10^{10}\left(E_{\mu} / 10^{19} \mathrm{eV}\right)$ $\mathrm{km}$, which implies that, in practice, the muon never decays inside the JEMEUSO field of view. 


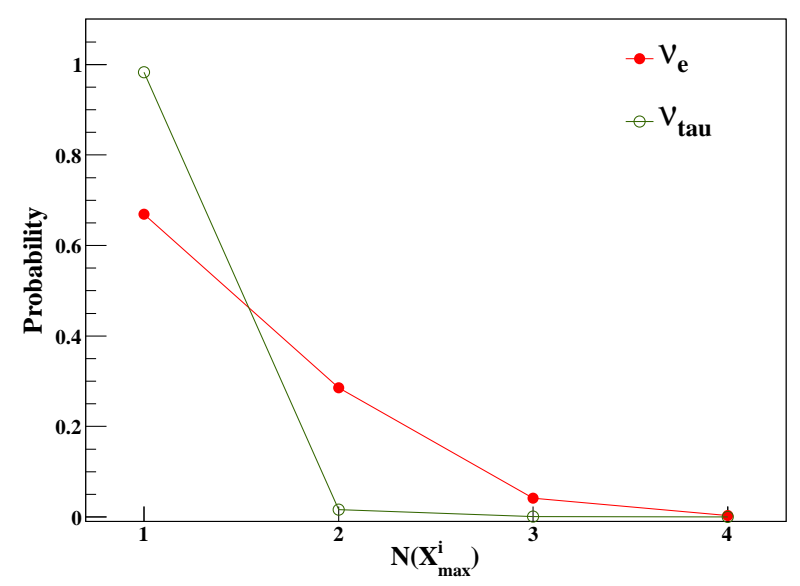

Fig. 7 Probability to find $N\left(X_{\max }^{i}\right)$ peaks in a profile corresponding to electron and tau neutrino showers whose first interaction point is at sea level on the vertical axis of JEMEUSO in Nadir mode. The neutrino energy is $E_{\nu}=10^{20} \mathrm{eV}$.

\subsection{Neutrino showers observed with JEM-EUSO}

The EUSO Simulation and Analysis Framework (ESAF) is used to simulate the response of JEM-EUSO to neutrino induced showers (see Ref. [41] for details). Figure 8 shows the time distributions of fluorescence photons that produce a signal on the photomultipliers (PMTs) for two events corresponding to horizontal electron neutrino showers of $10^{20} \mathrm{eV}$. The first interaction point is at sea level on the vertical axis of the JEM-EUSO telescope in Nadir mode. The shower corresponding to the event in the left panel has just one peak whereas the one on the right has two peaks. As can be seen from the figure, these characteristics are reflected in the distributions of detected photons. Note that the space between the photomultipliers can produce fake peaks specially for the cases in which there are few photoelectrons and the fluctuations are larger [42]. This is the case of the event on the right panel of the figure in which the second peak is divided into two. However, this fake peaks are easy to recognize because the configuration of the photomultipliers is known. The number of fluorescence photons that produced a signal is $\sim 228$ on average.

Figure 9 shows two simulated events under the same conditions as the ones corresponding to figure 8 except that the altitude is now set to $5 \mathrm{~km}$. It can be seen that the number of photons that produce signal on the PMTs increases and the time distributions become wider. The increase on the width of the time distributions of photons has to do with the fact that the showers develop closer to the telescope. Also the number of photons increases for higher altitudes due to this fact, however the dominant effect in the increase of the number of photons is the reduction in the attenuation suffered by the photons that propagate in the atmosphere from the shower track to the telescope. Note that also in this case the multi-peak structure of the showers can be observed in the distribution of photons detected by the telescope (right panel). In this 

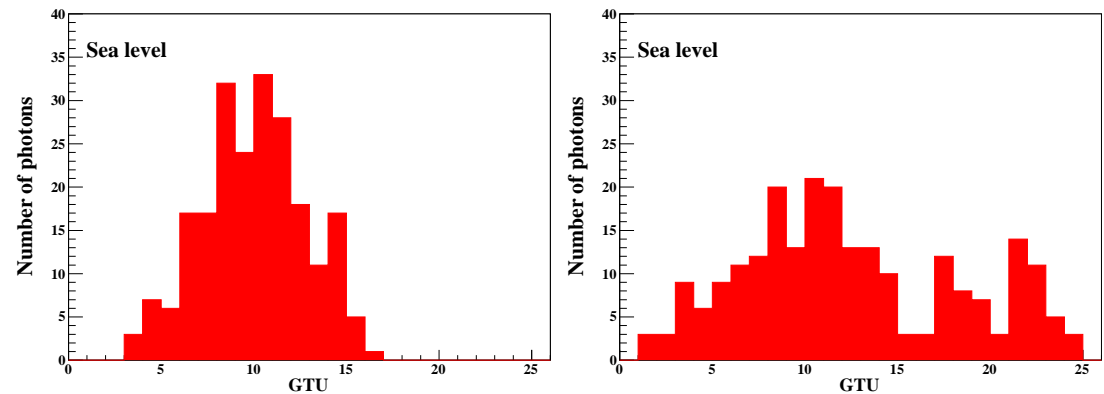

Fig. 8 Time distribution of fluorescence photons that produce signal on the PMTs for two electron neutrino horizontal showers of $E_{\nu}=10^{20} \mathrm{eV}$. The interaction point is at sea level in the center of the field of view of the JEM-EUSO telescope. The time is measured in Gate Time Units (GTUs), 1 GTU $=2.5 \mu \mathrm{s}$.

case the number of fluorescence photons contributing to the signal is $\sim 663$ on average; at $10 \mathrm{~km}$ this number increases to $\sim 1426$.
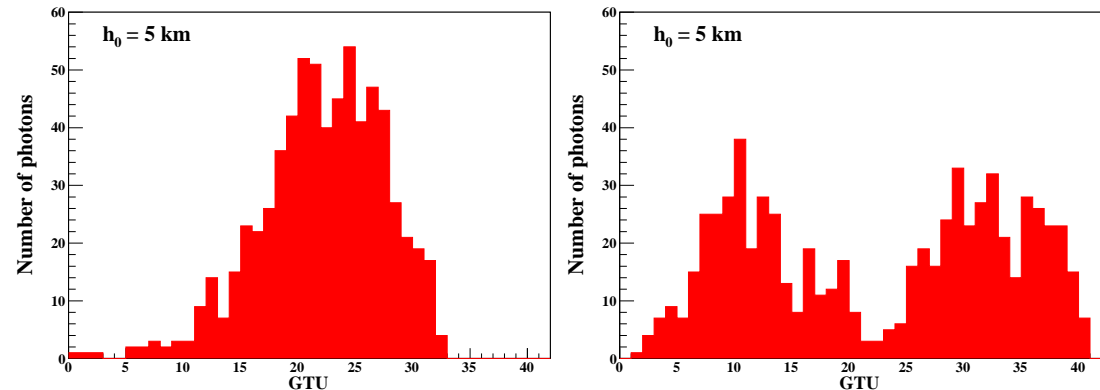

Fig. 9 Time distribution of photons that produce signal on the PMTs for two electron neutrino horizontal showers of $E_{\nu}=10^{20} \mathrm{eV}$. The interaction point is at an altitude of $h_{0}=5 \mathrm{~km}$ in the center of the field of view of the JEM-EUSO telescope.

Considering an isotropic flux of neutrinos with trajectories intersecting the ground, the maximum number of neutrino showers is achieved at a zenith angle of approximately $80^{\circ 1}$. Figure 10 shows the trigger efficiency of the JEM-EUSO telescope in Nadir mode as a function of the atmospheric depth of the first interaction point of the electron neutrino for the case of showers at $\theta=80^{\circ}$ that hit the ground on the center of the telescope's field of view. The first interaction point of the neutrino showers used for the calculation is selected at random from a uniform distribution in the interval $\left[0, X_{L}\left(80^{\circ}\right)\right]$, where $X_{L}\left(80^{\circ}\right) \cong 5766 \mathrm{~g} \mathrm{~cm}^{-2}$ is the grammage between the top of the atmosphere and the Earth's surface for $\theta=80^{\circ}$. The electron neutrino energies considered are $10^{20}, 10^{19.75}, 10^{19.5}$, and $10^{19.3} \mathrm{eV}$.

\footnotetext{
1 The zenith angle $\theta$ for which the maximum number of neutrino showers is achieved corresponds to the maximum of the product of the $2 \sin \theta \cos \theta$ distribution with the probability that a neutrino interacts in the atmosphere which is a function of $\theta$.
} 


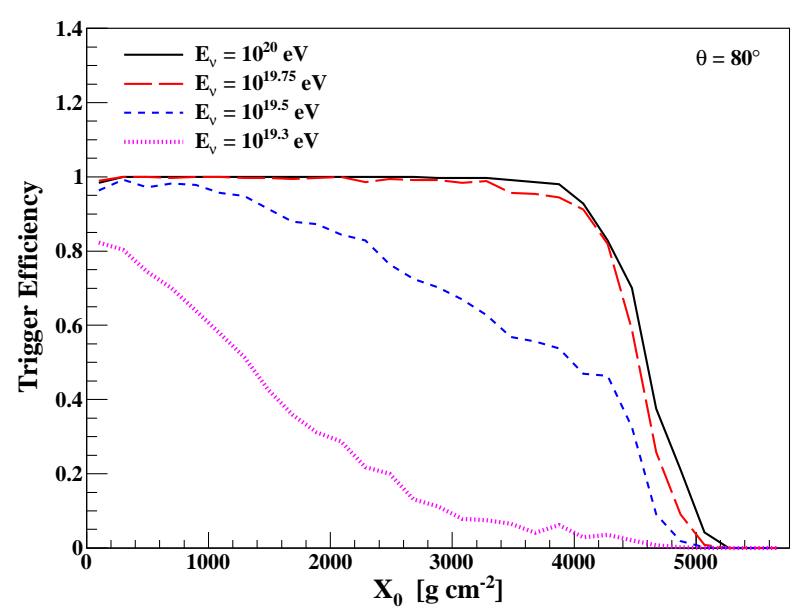

Fig. 10 Trigger efficiency as a function of the grammage of the first interaction point corresponding to electron neutrino showers that hit the ground on the center of the field of view of the JEM-EUSO telescope in Nadir mode. The zenith angle of the showers is $\theta=80^{\circ}$.

From figure 10 it can be seen that at $10^{20} \mathrm{eV}$ the trigger efficiency is approximately one form $X_{0}=0$ to $X_{0} \sim 4000 \mathrm{~g} \mathrm{~cm}^{-2}$, after that it starts to decrease reaching zero at the Earth's surface. The behavior of trigger efficiency close to the Earth's surface can be explained by the fact that the showers do not have time to develop completely before hitting the ground. For lower energies the deposited energy of the showers is smaller and then the number of fluorescence and Cherenkov photons generated during the shower development decreases producing smaller values of the trigger efficiency. For $10^{19.75} \mathrm{eV}$ the behavior of the trigger efficiency is similar to the one corresponding to $10^{20} \mathrm{eV}$ but it is smaller specially in the region close to the Earth's surface. Note that for smaller energies, $10^{19.5}$ and $10^{19.3} \mathrm{eV}$, the trigger efficiency is a decreasing function of $X_{0}$. This is due to the fact that for larger values of $X_{0}$ the showers are more affected by the LPM effect which makes the longitudinal profiles to be wider and smaller decreasing the number of triggered events.

\section{Photons}

\subsection{Characteristics of photon showers}

Ultra high energy photons can interact with the magnetic field of the Earth producing electron positron pairs, thus modifying the development of photon initiated atmospheric showers (see Ref. [43] for a review). The probability of this pre-showering depends on the intensity of the component of the magnetic field perpendicular to the direction of propagation of the photon. Thus, the characteristics of the corresponding showers strongly depend on the geographical position of the impact point and the direction of incidence of the 
incoming primary photon. Additionally, the development of a photon shower is influenced by the LPM effect which causes a reduction in pair production cross-section, retarding the development of the shower. The longitudinal evolution of high energy photon showers is dominated by the interplay between these two effects, with consequences for the possibility of discrimination between photon and proton showers.

The program CONEX is used to generate proton and photon showers. The impact points of the photon showers are distributed uniformly on the surface of the Earth in order to properly take into account pre-showering in the geomagnetic field. The arrival direction of the showers is distributed isotropically. The atmospheric depth corresponding to the point of maximum development of the cascades, $X_{\max }$, is one of the parameters most sensitive to the nature of the primary. $X_{\max }$ can be reconstructed from the future JEM-EUSO data and will play a fundamental role in the photon-hadron discrimination analyses.

Figure 11 shows, in Aitoff projection, a contour plot of $\operatorname{med}\left[X_{\max }^{\gamma}\right]-$ $\operatorname{med}\left[X_{\max }^{p r}\right]$ as a function of latitude and longitude of the impact point on the Earth for zenith angle $\theta \in\left[45^{\circ}, 90^{\circ}\right]$ and $E \in\left[10^{19.8}, 10^{20}\right] \mathrm{eV}$. Here med $\left[X_{\text {max }}^{A}\right]$ is the median of the distribution function of the shower maximum, $X_{\text {max }}^{A}$, for the particle type $A$ where $A=\gamma, p r$. It can be seen that there are regions over the Earth's surface where this difference is larger, resulting in more efficient discrimination between protons and photons. Note that the larger difference between the medians are located in the region of the South Atlantic Anomaly for which the intensity of the magnetic field is smaller.

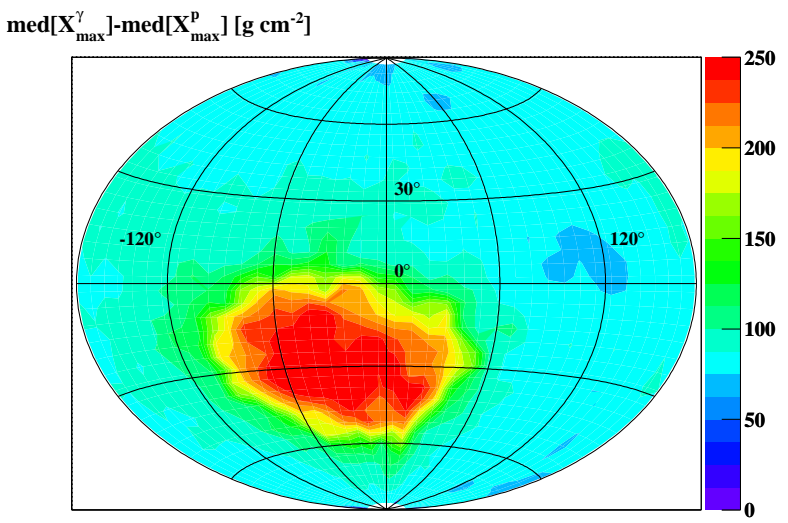

Fig. 11 Contour plot of med $\left[X_{\max }^{\gamma}\right]-\operatorname{med}\left[X_{\max }^{p r}\right]$ as a function of the latitude and longitude of the core location on the Earth surface. The showers considered are such that $\theta \in\left[45^{\circ}, 90^{\circ}\right]$ and $E \in\left[10^{19.8}, 10^{20}\right] \mathrm{eV}$. 
The discrimination power of the $X_{\max }$ parameter can be estimated through the merit factor,

$$
\eta=\frac{\operatorname{med}\left[X_{\max }^{\gamma}\right]-\operatorname{med}\left[X_{\max }^{p r}\right]}{\sqrt{\left(\Delta X_{\max }^{\gamma}\right)^{2}+\left(\Delta X_{\max }^{p r}\right)^{2}}},
$$

where $\Delta X_{\max }^{A}$ is one half of the length of the interval of $68 \%$ of probability of the $X_{\max }^{A}$ distribution. Events that satisfy a given cut in zenith angle and correspond to regions over the Earth surface for which $\eta \geq \eta_{\text {lim }}$, where $\eta_{\text {lim }}$ defines the minimum discrimination power, come from different regions of the universe. In particular, there are directions in the sky for which the discrimination between protons and photons is larger. Figure 12 shows a map in galactic coordinates of the distribution function of events that fulfill the following conditions: $\theta \in\left[45^{\circ}, 90^{\circ}\right], E \in\left[10^{19.8}, 10^{20}\right] \mathrm{eV}$, and $\eta_{\text {Lim }}=1.4$. A circular window of $5^{\circ}$ and a uniform exposure in right ascension are used for the calculation. Note that the regions on the sky where the distribution function

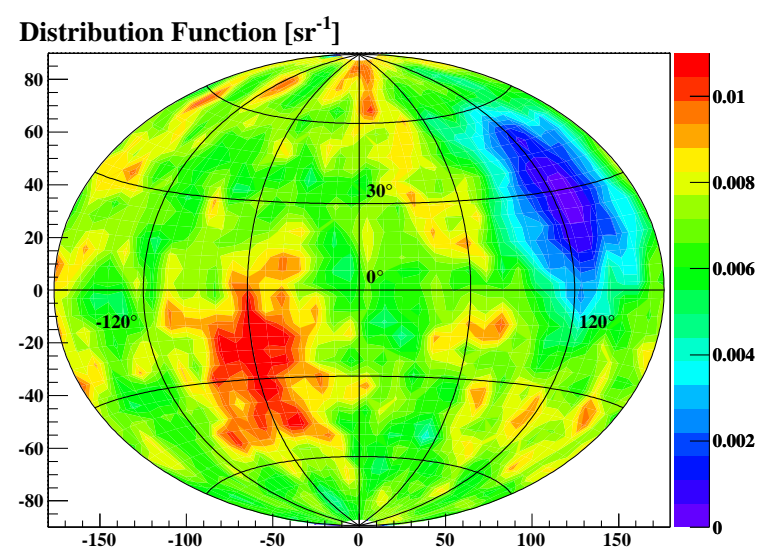

Fig. 12 Contour plot in galactic coordinates of the distribution function of the events with $\eta \geq 1.4, E \in\left[10^{19.8}, 10^{20}\right] \mathrm{eV}$, and $\theta \in\left[45^{\circ}, 90^{\circ}\right]$.

takes larger values correspond to regions on the Earth surface predominantly in the southern hemisphere where the maximum separation between protons and photons is obtained.

Figure 13 shows the $X_{\max }$ distribution for proton and photon primaries with $E \in\left[10^{19.8}, 10^{20}\right] \mathrm{eV}$ and $\theta \in\left[30^{\circ}, 60^{\circ}\right]$. It can be seen that the distribution function corresponding to photons has two populations, one corresponding to the photons pre-showered in the geomagnetic field and the one photons that do not pre-shower. Events that pre-shower exhibit smaller values of $X_{\max }$ and smaller fluctuations. Note that the presence of this population diminishes the discrimination power between photons and protons.

Figure 14 shows the medians and the regions of $68 \%$ probability of the $X_{\max }$ distribution for proton and photon primaries with $\theta \in\left[30^{\circ}, 60^{\circ}\right]$ as 


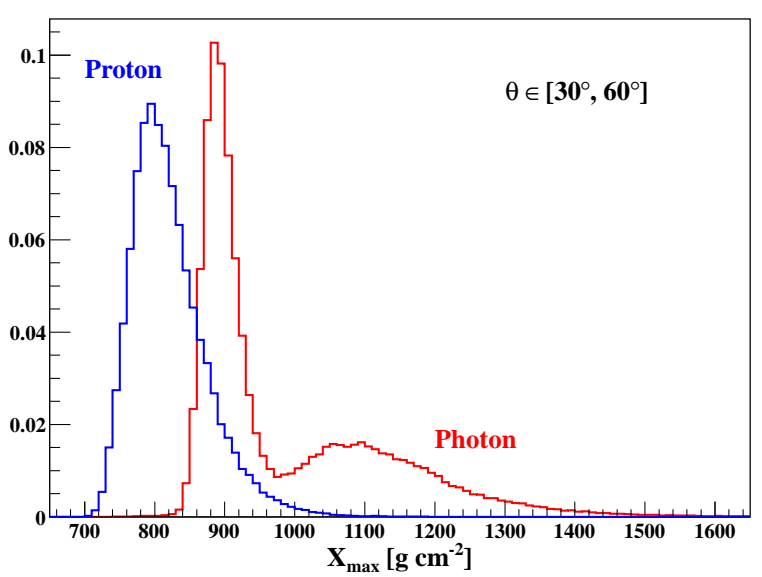

Fig. $13 X_{\max }$ distribution for proton and photon primaries with $E \in\left[10^{19.8}, 10^{20}\right] \mathrm{eV}$ and $\theta \in\left[30^{\circ}, 60^{\circ}\right]$. The histograms are normalized to unity.

a function of primary energy. It can be seen that for photon primaries the pre-showering effect starts to be important at energies larger than $\sim 10^{19.6}$ $\mathrm{eV}$. Between $\sim 10^{19.6}$ and $\sim 10^{20.1} \mathrm{eV}$ the $X_{\max }$ distribution is composed of both populations of events and for energies larger than $\sim 10^{20.1} \mathrm{eV}$ the $X_{\max }$ distribution is dominated by photons that pre-showered. Note that in the region dominated by the pre-showering effect the discrimination between protons and photons increases with primary energy, i.e. the separation between the medians and the regions of $68 \%$ probability of the proton and photon distributions increases with primary energy.

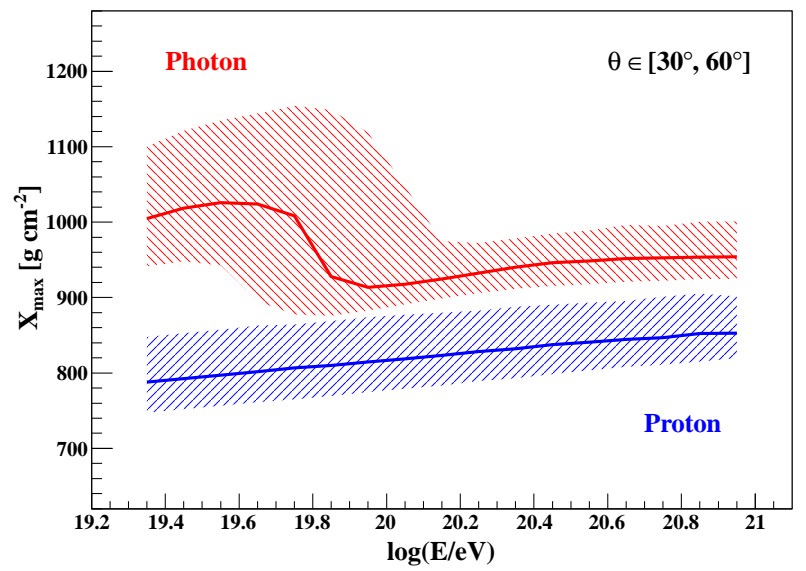

Fig. 14 Median and region of $68 \%$ probability of $X_{\max }$ as a function of primary energy for $\theta \in\left[30^{\circ}, 60^{\circ}\right]$. 


\subsection{Reach into photon fraction expected at JEM-EUSO}

Assuming that the JEM-EUSO exposure is the same as the one for protons [44] it is possible to calculate the expected number of photon events above a given energy threshold. Table 1 shows the expected number of photons with energies above $10^{19.6} \mathrm{eV}$, calculated considering the most optimistic photon flux taken from Ref. [2] (it corresponds to the curve on the top of the shadowed region of figure 15). The calculation is done for four cases: The observation of 5 and 10 years in the Nadir mode, 1 year in the Nadir mode and 4 years in the Tilted mode, and 1 year in the Nadir mode and 9 years in the Tilted mode. The tilted angle used for the calculation is $40^{\circ}$. Note that the number of events for the cases including observation in the Tilted mode the expected number of events is slightly smaller than the ones corresponding to observation in the Nadir mode. This is due to the fact that the exposure for $40^{\circ}$ of the Tilted angle is smaller than the one corresponding to the Nadir mode for energies higher than $\sim 10^{19.85} \mathrm{eV}$. For higher threshold energies this tendency is inverted.

\begin{tabular}{|c|c|c|c|}
\hline 5 yrs $N$ & 10 yrs $N$ & 1 yr $N+4$ yrs $T$ & 1 yr $N+9$ yrs $T$ \\
\hline$\sim 88$ & $\sim 177$ & $\sim 85$ & $\sim 168$ \\
\hline
\end{tabular}

Table 1 Expected number of photon events for energies larger than $10^{19.6} \mathrm{eV} . N$ corresponds to the Nadir mode of observation and $T$ to the Tilted one. The cosmogenic photon flux corresponds to the most optimistic case taken from Ref. [2]. The exposure used for the calculation is the one calculated for proton primaries.

Nevertheless, there are astrophysical models that predict a much smaller flux of cosmogenic photons, specially the ones that includes heavier nuclei in the composition injected by the sources (see for instance Ref. [3]). Also, fluctuations of a relatively large flux can still produce a null detection even for a non-null statistical expectation. For these reasons and also to compare with the existing upper limits on photon fractions obtained by different experiments the case in which there are no photons in the samples is studied below.

Let us consider the ideal situation in which it is known that there are no photons in a given sample of $N$ events, i.e. it is possible to separate hadrons from photons in an event-by-event basis. In this case, the expression for the upper limit on the photon fraction is given by [43],

$$
\mathcal{F}_{\gamma}^{\text {min }}=1-(1-\alpha)^{1 / N}
$$

where $\alpha$ is the confidence level of rejection. In practice, however, the probability of the existence of photons must be assessed through some observational technique, which leads unavoidably to less restrictive upper limits than in the ideal case. 
The method used to calculate the upper limit from $X_{\max }$ measurements is based on the abundance estimator first introduced in [45],

$$
\xi_{X_{\max }}=\frac{1}{N} \sum_{i=1}^{N} \frac{f_{\gamma}\left(X_{\max }^{i}\right)}{f_{\gamma}\left(X_{\max }^{i}\right)+f_{p r}\left(X_{\max }^{i}\right)}
$$

where $f_{\gamma}\left(X_{\max }\right)$ and $f_{p r}\left(X_{\max }\right)$ are the photon and proton distribution functions, $X_{\max }^{i}$ are experimental values of $X_{\max } . \xi_{X_{\max }}$ is an estimator of the photon abundance.

For the case in which the sample size is large enough it is possible to analytically calculate an upper limit on the photon fraction assuming that $\xi_{X_{\max }}$ is a Gaussian variable [46]. In this work the expected upper limits on the photon fraction is calculated using a Monte Carlo technique which is valid for samples of all sizes. The distribution functions needed to calculate $\xi_{X_{\max }}$ are obtained from the simulated data by using the non-parametric method of kernel superposition with adaptive bandwidth $[47,45]$. The number of events expected above a given energy threshold are calculated by using a brokenpower law fit of the Auger energy spectrum [55] and the exposure of JEMEUSO [44].

Figure 15 shows the upper limits on the photon fraction in the integral flux at $95 \%$ confidence level as a function of primary energy. In the calculation it is assumed that there are no photons in the samples. The zenith angle of the showers is in the interval $\left[45^{\circ}, 90^{\circ}\right]$ and a Gaussian uncertainty on the determination of $X_{\max }$ of $100 \mathrm{~g} \mathrm{~cm}^{-2}$ is assumed. The solid lines show the expected upper limits for the case in which the observation is performed in Nadir mode for 5 and 10 years. The dot-dash-dot lines correspond to the observation of one year in Nadir mode and 4 and 9 years in Tilted mode for 5 and 10 years of total observation time, respectively. A Tilted angle of $40^{\circ}$ is used for the calculation. The curves corresponding to the ideal case in which it is possible to discriminate between photons and protons in an event-by-event basis are also shown.

Although the expected upper limits are smaller than the existing ones, even more restrictive upper limits can be obtained by improving discrimination between protons and photons. As seen from figure 15, the expected upper limits for the ideal case described by Eq. (4) are below those obtained by using the $\xi_{X_{\max }}$ method, showing that there is still potential room for improvement.

\section{Conclusions}

In this work we have studied the characteristics of the longitudinal profiles of the air showers initiated by ultra high energy photons and neutrinos which can be employed to identify these types of primaries. We have shown that very deep horizontal neutrino showers produce a multi-peak structure that can be observed in the data recorded by the JEM-EUSO telescope. We have also discussed the possibility of discriminating between electron and tau neutrinos 


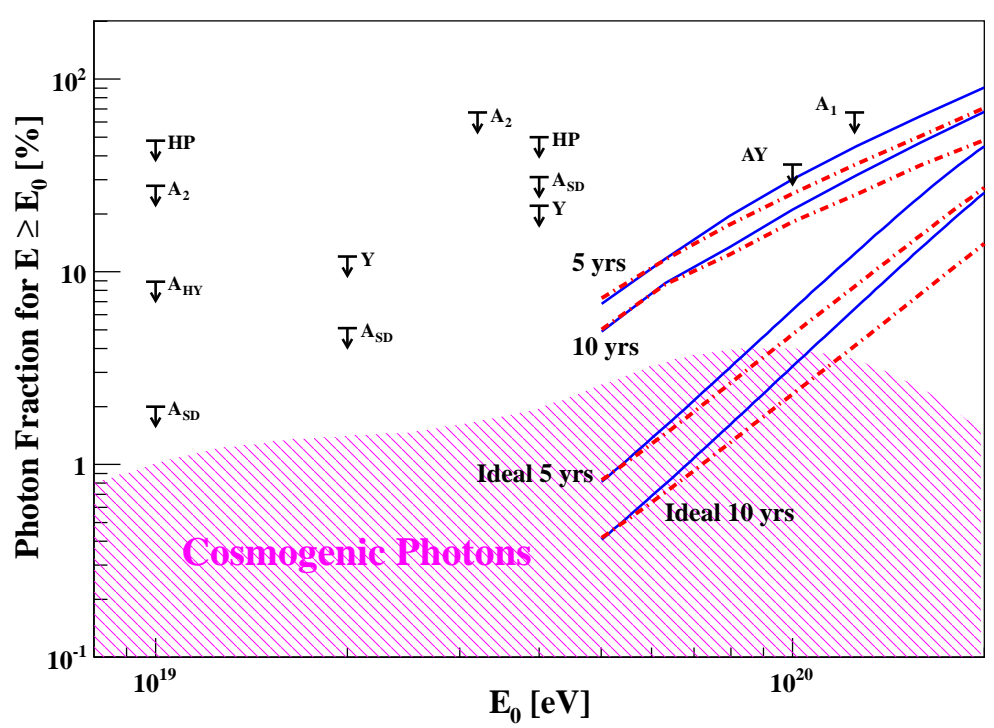

Fig. 15 Upper limits on the fraction of photons in the integral cosmic ray flux at $95 \%$ confidence level as a function of primary energy. Solid lines correspond to the expected upper limits for JEM-EUSO in Nadir mode and dot-dash-dot lines correspond to a combination of Nadir and Tilted modes (see text). A Gaussian uncertainty on the determination of the $X_{\max }$ parameter of $100 \mathrm{~g} \mathrm{~cm}^{-2}$ is assumed for the calculations. The shaded region indicates the prediction for GZK photons [2]. Black arrows are experimental limits, HP: Haverah Park [48]; $\mathrm{A}_{1}, \mathrm{~A}_{2}$ : AGASA [49,50]; $\mathrm{A}_{\mathrm{HY}}, \mathrm{A}_{\mathrm{SD}}$ : Auger [51,52]; AY: AGASA-Yakutsk [53]; Y: Yakutsk [54].

by taking advantage of the dominant hadronic component of tau neutrino showers. Finally, we have calculated the expected upper limits on the photon fraction in the integral cosmic ray flux expected by JEM-EUSO. We have shown that it is possible to improve the existing upper limits at the highest energies. In any case, there is still room to improve the methods proposed here in order to make a discovery or improve upper limits.

\section{References}

1. Gelmini, G., Kalashev, O., and Semikoz, D., GZK Photons in the Minimal Ultrahigh Energy Cosmic Rays Model, Astropart. Phys., 28, 390 (2007).

2. Gelmini, G., Kalashev O., and Semikoz, D., GZK Photons Above 10-EeV, JCAP, 0711, $002(2007)$

3. Hooper, D., Taylor, A. M., and Sarkar, S., Cosmogenic photons as a test of ultra-high energy cosmic ray composition, Astropart. Phys., 34, 340 (2011).

4. Beresinsky, V. and Zatsepin, G., Cosmic rays at ultrahigh-energies (neutrino?), Phys. Lett. B, 28, 423 (1969).

5. Stecker, F., Diffuse Fluxes Of Cosmic High-Energy Neutrinos, Astrophys. J., 228, 919 (1979).

6. Yoshida, S. and Teshima, M., Energy spectrum of ultrahigh-energy cosmic rays with extragalactic origin, Prog. Theor. Phys., 89, 833 (1993).

7. Protheroe, R. and Johnson, P., Propagation of ultrahigh-energy protons over cosmological distances and implications for topological defect models, Astropart. Phys., 4, 253 (1996). 
8. Engel, R., Seckel, D., and Stanev, T., Neutrinos from propagation of ultra-high energy protons, Phys. Rev. D, 64, 093010 (2001).

9. Fodor, Z., Katz, S., Ringwald, A., and Tu, H., Bounds on the cosmogenic neutrino flux, JCAP, 0311, 015 (2003).

10. Anchordoqui, L., Goldberg, H., Hooper, D., Sarkar, S., and Taylor, A. M., Predictions for the cosmogenic neutrino flux in light of new data from the Pierre Auger Observatory, Phys. Rev. D, 76, 123008 (2007).

11. Kotera, K., Allard, D., and Olinto, A., Cosmogenic neutrinos: parameter space and detectabilty from $\mathrm{PeV}$ to ZeV, JCAP, 1010, 013 (2010).

12. Ahlers, M. and Halzen, F., Minimal Cosmogenic Neutrinos, [arXiv:1208.4181 [astroph.HE]].

13. Hooper, D., Taylor, A., and Sarkar, S., The Impact of heavy nuclei on the cosmogenic neutrino flux, Astropart. Phys., 23, 11 (2005).

14. Ave, M., Busca, N., Olinto, A., Watson, A., and Yamamoto, T., Cosmogenic neutrinos from ultra-high energy nuclei, Astropart. Phys., 23, 19 (2005).

15. Gaisser, T., Halzen, F., and Stanev, T., Particle astrophysics with high-energy neutrinos, Phys. Rept., 258, 173 (1995). [Erratum-ibid., 271, 355 (1996)]

16. Halzen, F. and Hooper, D., High-energy neutrino astronomy: The Cosmic ray connection, Rept. Prog. Phys., 65, 1025 (2002).

17. J. K. Becker, Phys. Rept. 458, 173 (2008) [arXiv:0710.1557 [astro-ph]].

18. Anchordoqui, L. and Montaruli, T., In Search for Extraterrestrial High Energy Neutrinos, Ann. Rev. Nucl. Part. Sci., 60, 129 (2010).

19. Kuzmin, V. and Tkachev, I., Ultrahigh-energy cosmic rays and inflation relics, Phys. Rept., 320, 199 (1999).

20. Bhattacharjee, P. and Sigl, G., Origin and propagation of extremely high-energy cosmic rays, Phys. Rept., 327, 109 (2000).

21. Berezinsky, V., Sabancilar, E., and Vilenkin, A., Extremely High Energy Neutrinos from Cosmic Strings, Phys. Rev. D, 84, 085006 (2011).

22. Whitehorn, N., Results from IceCube, talk given at IceCube Particle Astrophysics Symposium (IPA 2013), Madison WI, May 13 -15, 2013. http://wipac.wisc.edu/meetings/home/IPA2013.

23. Kashti, T. and Waxman, E., Phys. Rev. Lett., 95, 181101 (2005).

24. Lipari, P., Lusignoli, M., and Meloni, D., Phys. Rev. D, 75, 123005 (2007)

25. Hümmer, S, . et al., Astropart. Phys., 34, 205 (2010).

26. Pakvasa, S., Mod. Phys. Lett., A23, 1313 (2008).

27. Blennow, M. and Meloni, D., Phys. Rev., D80, 065009 (2009).

28. Beacom, J., Phys. Rev., D68, 093005 (2003); Erratum-ibid., D72, 019901 (2005).

29. Block, M., Ha, P., and McKay, D., Ultrahigh energy neutrino scattering: An update Phys. Rev. D, 82, 077302 (2010).

30. Sjöstrand, T., Mrenna, S., and Skands, P., PYTHIA 6.4 physics and manual, JHEP, 0605, 026 (2006).

31. M. Whalley et al., http://hepforge.cedar.ac.uk/lhapdf/.

32. Nadolsky, P. et al., Implications of CTEQ global analysis for collider observables, Phys. Rev. D, 78, 013004 (2008).

33. Supanitsky, A. D. and Medina-Tanco, G., Neutrino initiated cascades at mid and high altitudes in the atmosphere, Astropart. Phys., 34, 8-16 (2011).

34. T. Bergmann et al., One-dimensional hybrid approach to extensive air shower simulation, Astropart. Phys., 26, 420-432 (2007).

35. S. Ostapchenko, QGSJET-II: towards reliable description of very high energy hadronic interactions, Nucl. Phys. Proc. Suppl. B, 151, 143-146 (2006).

36. Landau, L. and Pomeranchuk, I., Limits of applicability of the theory of bremsstrahlung electrons and pair production at high-energies, Dokl. Akad. Nauk SSSR, 92, 535-536 (1953).

37. Landau, L. and Pomeranchuk, I., Electron cascade process at very high-energies, Dokl. Akad. Nauk SSSR, 92, 735-738 (1953).

38. Migdal, A., Bremsstrahlung and pair production in condensed media at high-energies, Phys. Rev., 103, 1811-1820 (1956).

39. Supanitsky, A. D. and Medina-Tanco, G., On the possibility of neutrino flavor identification at the highest energies, Phys. Rev. D, 86, 093020 (2012). 
40. Davidson, N., Nanava, G., Przedziński, T., Richter-Was, E., and Was, Z., Universal interface of TAUOLA: Technical and physics documentation, Comput. Phys. Commun. 183, 821-843 (2012).

41. Berat, C., et al., Full simulation of space-based extensive air showers detectors with ESAF, Astropart. Phys., 33, 221-247 (2010).

42. Guzman, A. et al., A study on JEM-EUSOs trigger probability for neutrino-initiated EAS, Proceedings of the 33rd ICRC, Brazil, \# 533 (2013).

43. Risse, M. and Homola, P., Search for Ultra-High Energy Photons Using Air Showers, Mod. Phys. Lett., A22, 749-766 (2007).

44. Adams Jr., J.H. et al. (JEM-EUSO Collaboration), An evaluation of the exposure in nadir observation of the JEM-EUSO mission, Astropart. Phys., 44, 76-90 (2013).

45. Supanitsky, A. D., Medina-Tanco, G., and Etchegoyen, A new numerical technique to determine primary cosmic ray composition in the ankle region, A., Astropart. Phys., 31, 75-85 (2009).

46. Supanitsky, A. D. and Medina-Tanco, G. et al. (JEM-EUSO Collaboration), The potential of the JEM-EUSO telescope for the astrophysics of extreme energy photons, Proceedings of the 32nd ICRC, Beijing, vol. 2, 153-156 (2011).

47. Silvermann, B., Density Estimation for Statistics and Data Analysis. Chapman and Hall, London (1986).

48. Ave M. et al., New Constraints from Haverah Park Data on the Photon and Iron Fluxes of Ultrahigh-Energy Cosmic Rays, Phys. Rev. Lett., 85, 2244-2247 (2000). Ave M. et al., Constraints on the ultrahigh-energy photon flux using inclined showers from the Haverah Park array, Phys. Rev. D, 65, 063007 (2002).

49. Risse, M. et al., Upper limit on the photon fraction in highest-energy cosmic rays from AGASA data, Phys. Rev. Lett., 95, 171102 (2005).

50. Shinozaki, K. et al., Upper limit on gamma-ray flux above $10^{19} \mathrm{eV}$ estimated by the Akeno Giant Air Shower Array, Astrophys. J., 571, L117-L120 (2002).

51. J. Abraham et al. (Pierre Auger Collaboration), An upper limit to the photon fraction in cosmic rays above $10^{19} \mathrm{eV}$ from the Pierre Auger Observatory, Astropart. Phys., 27, 155-168 (2007). Settimo, M. et al. (Pierre Auger Collaboration), An update on a search for ultra-high energy photons using the Pierre Auger Observatory, Proceedings of the 32nd ICRC, Beijing, vol. 2, 55-58 (2011).

52. J. Abraham et al. (Pierre Auger Collaboration), Upper limit on the cosmic-ray photon flux above $10^{19} \mathrm{eV}$ using the surface detector of the Pierre Auger Observatory, Astropart. Phys., 29, 243-256 (2008).

53. Rubtsov, G. et al., Upper limit on the ultrahigh-energy photon flux from AGASA and Yakutsk data, Phys. Rev. D, 73, 063009 (2006).

54. A. Glushkov et al., Constraints on the fraction of primary gamma rays at ultra-high energies from the muon data of the Yakutsk EAS array, JETP Lett., 85, 131-135 (2007).

55. Salamida, F. et al. (Pierre Auger Collaboration), Update on the measurement of the CR energy spectrum above $10^{18} \mathrm{eV}$ made using the Pierre Auger Observatory, Proceedings of the 32nd ICRC, Beijing, vol. 2, 145-148 (2011). 